\title{
Creative Music Therapy in an Acute Care Setting for Older Patients with Delirium and Dementia
}

\author{
Chin Yee Cheong Jane An Qi Tan Yi-Lin Foong Hui Mien Koh \\ Denise Zhen Yue Chen Jessie Joon Chen Tan Chong Jin Ng Philip Yap
}

Department of Geriatric Medicine, Khoo Teck Puat Hospital, Singapore, Singapore

\section{Key Words}

Music therapy $\cdot$ Acute hospital $\cdot$ Engagement $\cdot$ Delirium $\cdot$ Dementia

\begin{abstract}
Background/Aims: The acute hospital ward can be unfamiliar and stressful for older patients with impaired cognition, rendering them prone to agitation and resistive to care. Extant literature shows that music therapy can enhance engagement and mood, thereby ameliorating agitated behaviours. This pilot study evaluates the impact of a creative music therapy (CMT) programme on mood and engagement in older patients with delirium and/or dementia (PtDD) in an acute care setting. We hypothesize that CMT improves engagement and pleasure in these patients. Methods: Twenty-five PtDD (age 86.5 \pm 5.7 years, MMSE 6/30 \pm 5.4 ) were observed for $90 \mathrm{~min}$ (30 min before, 30 min during, and 30 min after music therapy) on 3 consecutive days: day 1 (control condition without music) and days 2 and 3 (with CMT). Music interventions included music improvisation such as spontaneous music making and playing familiar songs of patient's choice. The main outcome measures were mood and engagement assessed with the Menorah Park Engagement Scale (MPES) and Observed Emotion Rating Scale (OERS). Results: Wilcoxon signed-rank test showed a statistically significant positive change in constructive and passive engagement $(Z=3.383, p=0.01)$ in MPES and pleasure and general alertness $(Z=3.188, p=0.01)$ in OERS during CMT. The average pleasure ratings of days 2 and 3 were higher than those of day $1(Z=2.466, p=0.014)$. Negative engagement $(Z=2.582, p=0.01)$ and affect $(Z=2.004, p=0.045)$ were both lower during CMT compared to no music. Conclusion: These results suggest that CMT holds much promise to improve mood and engagement of PtDD in an acute hospital setting. CMT can also be scheduled into the patients' daily routines or incorporated into other areas of care to increase patient compliance and cooperation.




\section{Introduction}

Individuals with dementia have increased rates of hospitalizations and poorer outcomes compared to their counterparts without dementia [1]. The prevalence of dementia among hospitalized patients ranges from 8 to $27 \%$ [2-4]. A diagnosis of dementia is associated with both a longer length of stay and higher per capita cost, mostly due to deterioration in cognitive and physical function during hospitalization [5-7].

Conversely, delirium is an acute disorder of attention and cognition. It is characterized by disorganized thinking, fluctuating levels of alertness, disorientation, and disturbed psychomotor activity. Delirium is common among patients with dementia in acute care settings. The prevalence of delirium superimposed on dementia has been reported in $22 \%$ of hospitalized patients $\geq 65$ years of age [8]. A quarter of older patients with delirium die within 1 month of its onset with a recent study estimating that the risk of mortality increases by $11 \%$ for every additional $48 \mathrm{~h}$ of delirium $[9,10]$. It is often overlooked because of its non-specific presentation and is commonly misdiagnosed as depression or psychosis.

The care of patients with delirium and/or dementia (PtDD), in particular those with disruptive behaviours, poses significant challenges to health professionals and hospitals. Not uncommonly, these patients are subjected to psychotropic medications and physical restraints in desperate efforts to control their disruptive behaviours. However, physical and chemical restraints both carry the risk of adverse effects [11]. Studies have evidenced physical restraints to increase the risk of death, falls, serious injury, and duration of hospitalization [12], while the higher mortality associated with psychotropic medications has been widely publicized in the past decade.

Consequently, alternative approaches to the care of PtDD to reduce the use of chemical or physical restraints are greatly needed. Thus, non-pharmacological multi-component approaches are increasingly accepted as effective strategies for disruptive behaviours [13]. They have been shown to significantly decrease agitation and increase pleasure and interest [14]. The most widely used interventions include facilitating orientation, pain relief, optimizing hydration, proactive and early mobilization, and active review by specialist nurses or geriatricians [15]. Disruptive behaviours can arise due to sensory deprivation and lack of meaningful engagement [16]. Music therapy, another non-pharmacological intervention, has been used to improve engagement and decrease agitated behaviours in individuals with dementia mostly in long-term care settings [17]. As such, it may hold promise as a non-pharmacological intervention for PtDD in acute care settings.

Music therapy is a goal-directed process in which the therapist helps the patient to improve, maintain, or restore a state of well-being. Despite the mechanisms of the effects of music therapy were not well understood, Hillecke et al. [18] presented a heuristic model, consisting of five working factors of music therapy (attention modulation, emotion modulation, cognition modulation, behaviour modulation, and communication modulation). Musical experiences and the relationship that develop through them are used as the main forces of change. It has been tried as a nursing intervention to decrease acute postoperative confusion and delirium in elderly undergoing elective hip and knee surgery [19] and to decrease anxiety and promote relaxation in patients receiving ventilator assistance, with consequent decrease in heart and respiratory rates [20].

Musical experiences used in the course of therapy include improvising, performing, composing, notating, verbalizing, and listening to music. There are many ways in which these can be designed and harnessed. In this study, the main approach used was the NordoffRobbins creative music therapy (CMT). It is a patient-centred, improvisational approach to individual and group music therapy based on the premise that every individual has an innate musicality and ability to respond to music [21, 22]. 
Cheong et al.: Creative Music Therapy in an Acute Care Setting for Older Patients with Delirium and Dementia

Table 1. Demographic and clinic characteristics of the study population $(n=25)$

$\begin{array}{lr}\text { Females } & 15(60) \\ \text { Chinese ethnicity } & 15(60) \\ \text { Mean age } \pm \text { SD, years } & 86.5 \pm 5.7 \\ \text { Mean MMSE } \pm \text { SD } & 6 \pm 5.4 \\ \text { Mean Charlson comorbidity index } \pm \text { SD } & 7.2 \pm 1.6 \\ \text { Mean Pittsburgh Agitation Scale on admission } \pm \text { SD } & 3.0 \pm 2.8 \\ \text { Type of dementia } & \\ \quad \text { Alzheimer's disease } & 9(36) \\ \text { Vascular dementia } & 9(36) \\ \text { Mixed dementia } & 5(20) \\ \text { Other type of dementia } & 1(4) \\ \text { No dementia } & 1(4) \\ \text { Delirium } & 8(32)\end{array}$

Values are n $(\%)$ or means \pm SD.

Although the quality of several earlier studies on music therapy for persons with dementia lacked methodological rigour in general, a meta-analysis concluded that music therapy held potential for increasing quality of life for patients with dementia [23]. A study by Takahashi et al. [22] showed long-term benefit of regular music therapy in maintaining physical and mental well-being of PtDD. Other studies have also demonstrated promising outcomes in improving agitation in individuals with dementia with preferred music [17, 24-27].

CMT is 'active' as the patient's music making and responses form the core of the therapeutic work. The 'creative' aspects lie in the music therapist creating and improvising music which is used to establish and maintain a connection with the patient. As music improvisation is a creative yet flexible process, CMT has the potential to transcend age and pathologies, verbal and functional abilities, possibly benefitting PtDD who have varying cognitive disabilities. Therefore, this study aims to examine the effects of CMT in improving well-being, particularly mood and engagement, of PtDD in an acute care hospital setting.

\section{Methods}

\section{Study Setting}

This pilot study was conducted over a 3-month period in a 10-bed acute care unit for PtDD in a geriatric medicine department of a tertiary hospital. PtDD were attending an on-going CMT programme run by a music therapist who is certified by the Certification Board for Music Therapists in the United States. Based on individual's profile and response to music, the music therapist modified and adapted techniques to meet the patients' needs. The relevant ethics approval (National Healthcare Group Domain Specific Review Board) had been obtained for the study to be conducted.

\section{Participants}

Twenty-five consecutive PtDD (mean age $86.5 \pm 5.7$ years, mean MMSE 6/30 \pm 5.4 ) who satisfied the inclusion criteria were recruited (table 1). The selection criteria comprised: (i) diagnosis of dementia with or without delirium, (ii) age $\geq 65$ years, (iii) no prior experience with CMT and willing to engage in music therapy intervention, (iv) absence of severe hearing impairment or able to hear using hearing aids, ( $v$ ) not suffering from severely disruptive and combative behaviours that impair attention and engagement, and (vi) not medically unstable. 
Table 2. Time frame for mapping

\begin{tabular}{lll}
\hline Time & Day 1 & Days 2 and 3 \\
\hline 09:30 & $\begin{array}{l}\text { Subjects as controls observed for 30 min } \\
\text { without music intervention }\end{array}$ & $\begin{array}{l}\text { Subjects observed for 30 min without music } \\
\text { intervention }\end{array}$ \\
\hline 10:00 & $\begin{array}{l}\text { Subjects as controls observed for 30 min } \\
\text { without music intervention }\end{array}$ & $\begin{array}{l}\text { 30 min of CMT in group setting or one-to-one } \\
\text { sessions involving: } \\
\text { (1) Clinical music improvisation, e.g. spontaneous } \\
\text { music making with musical instruments with } \\
\text { therapist on the keyboard/guitar }\end{array}$ \\
& & $\begin{array}{l}\text { (2) Playing familiar songs of patient's choice } \\
\text { (3) Music listening }\end{array}$ \\
& & Subjects observed for 30 min after CMT \\
\hline $10: 30$ & Subjects as controls observed for 30 min & \\
& without music intervention & \\
\hline
\end{tabular}

Dementia was diagnosed by geriatricians according to the Diagnostic and Statistical Manual of Mental Disorders IV (DSM-IV) [28], while delirium was ascertained with the Confusion Assessment Method (CAM) [29]. Screening of candidates suitable for CMT was done upon admission to the unit. CMT was typically introduced on day 2 of admission after the initial medical evaluation.

\section{Instruments and Data Collection}

Direct observation instruments were utilized to assess engagement and affect. Observers were trained using an established protocol to ensure inter-rater reliability. The Menorah Park Engagement Scale (MPES) [30], which was initially developed to assess levels of engagement in adult day care patients during Montessori-based dementia programming, was used to assess engagement. It captures four types of engagement: constructive engagement, passive engagement, self-engagement, and non-engagement.

Constructive engagement referred to any motor or verbal behaviour exhibited in response to the activity the patient was participating in. Passive engagement pertained to listening and/or observing in response to the activity presented. Self-engagement was defined as any purposeless behaviour involving the patient's engagement with himself or herself during the activity. Non-engagement related to staring into space or another direction away from the activity for $>10 \mathrm{~s}$ or sleeping during an activity. The duration of each type of engagement was recorded in 5 -min periods. If $\geq 2$ types of engagement took place during a 5-min interval, raters made coding decisions based on a hierarchical ladder in which the behaviour most indicative of the main form of engagement was chosen so that only one behaviour for each 5-min interval was coded.

The Lawton Observed Emotion Rating Scale (OERS), a standardized and validated instrument, was used to measure affect $[31,32]$. The scale was initially developed to evaluate patients with Alzheimer's disease in nursing homes. It has a high reliability (kappa 0.76-0.89) [32]. The five types of emotion recorded included: pleasure, general alertness, anger, anxiety/ fear, and sadness. Observers used standardized guidelines on facial and vocal expressions to code the different categories of emotion. PtDD were observed and rated during the same time period from 09:30 to 11:00 on both music intervention days (days 2 and 3) and on days with the control condition (day 1) (table 2). Two raters, who coded engagement and affect independently, were deployed for every observation so that the patients' affect and engagement were both assessed during each time frame. The two raters would compare their ratings after 
Cheong et al.: Creative Music Therapy in an Acute Care Setting for Older Patients with Delirium and Dementia

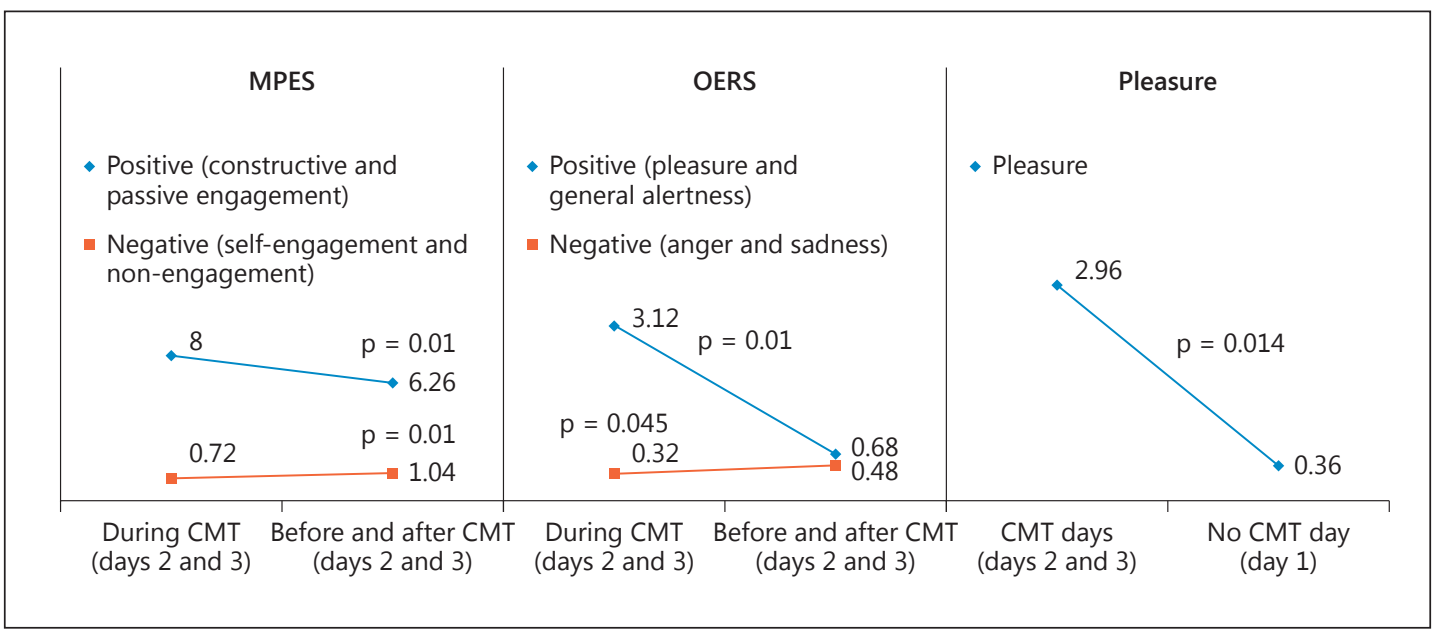

Fig. 1. Mean frequency of the MPES and OERS.

each session and where differences arose; a consensus on the final rating would be reached. A within-subject design, whereby PtDD acted as their own controls, was adopted.

On day 1, patients were mapped for 90 min during which they would be receiving usual care such as assistance in personal care and hygiene, measurements of vital signs, therapy sessions with occupational therapists or physiotherapists, or doctors' rounds. On days 2 and 3 , patients were mapped 30 min before, during, and after the music therapy session. Patients would have been receiving usual care before the music therapy session, which typically lasted 30 min, and would continue with usual care afterwards. A board certified music therapist conducted the CMT sessions for all patients. The music therapist engaged the patient in both active music making and/or music listening and worked toward building a work of aesthetic value by embracing whatever musical material the patient offered.

\section{Quantitative and Statistical Analysis}

The MPES and Lawton OERS were rated for each patient with 5-min intervals. One point was accorded for the behaviour most frequently observed in each scale during the 5-min interval. The mean scores on the MPES and Lawton OERS were then obtained for the 30-min time frame before, during, and after the music therapy session. The non-parametric Wilcoxon signed-rank test was used to analyze the mean difference in engagement and affect between the different time periods (CMT session versus before and after CMT) on a day with CMT, and between days with CMT (days 2 and 3) and days without CMT (day 1). All analyses were performed using SPSS version 20.

\section{Results}

\section{Engagement}

The frequency of positive MPES (constructive and passive engagement) showed statistically significant higher occurrences during the CMT session (days 2 and 3 ; mean $=8.00$ ) compared to before and after the CMT sessions (mean $=6.26, \mathrm{Z}=3.383, \mathrm{p}=0.01$ ). Whereas for negative MPES (self-engagement and non-engagement), the frequency was statistically lower during the CMT session (days 2 and 3; mean $=0.72$ ) compared to before and after the CMT sessions (mean $=1.04, \mathrm{Z}=2.582, \mathrm{p}=0.01$ ) (fig. 1 ). 
Cheong et al.: Creative Music Therapy in an Acute Care Setting for Older Patients with Delirium and Dementia

\section{Mood/Emotion}

Positive OERS (pleasure and general alertness) had higher occurrences during the CMT sessions (days 2 and 3; mean $=3.12$ ) compared to before and after CMT (mean $=0.68, \mathrm{Z}=$ $3.188, \mathrm{p}=0.01$ ). The frequency of negative OERS observances (anger, anxiety, and sadness) was statistically lower during the CMT sessions (days 2 and 3 ; mean $=0.32$ ) compared to before and after the CMT sessions (mean $=0.48, \mathrm{Z}=2.004, \mathrm{p}=0.045$ ). Item level analysis of OERS showed statistically significant positive change in the pleasure component during CMT days (days 2 and 3; mean $=2.96$ ) compared to the day without CMT (day 1 ; mean $=0.36, \mathrm{Z}=$ $2.466,167 \mathrm{p}=0.014)$.

\section{Discussion}

The findings contribute to the growing corpus of knowledge on the efficacy of music therapy in patients with dementia. Previous studies, typically in long-term care settings, have focused mainly on the overall effects of music therapy over a more extended period of time [22]. This is, however, less relevant in the acute hospital setting where the length of stay is usually in the range of days. The key finding that CMT has a more proximal effect on mood and engagement could indicate its role as an adjunct in facilitating treatments, such as administration of medication or wound dressings, and other forms of therapy in physiotherapy or occupational therapy. It is known that PtDD can be resistive to custodial care and nursing procedures and not uncommonly refuse participation in therapy. CMT can be incorporated in the daily care of such patients to improve patient compliance and cooperation. Johnson et al.'s [33] pilot study, for example, supported the use of music in improving exercise participation in patients with dementia.

CMT may exert its benefits in engagement and mood due to the effects of music on attention [34], general well-being, and quality of life. Familiar music from the past can assist in memory recall and elicit memories associated with positive feelings [35]. It may also redirect patient's attention from confusing stimuli and consequently ameliorate anxiety and agitation. Success in singing, playing instruments, moving to music, or sharing memories related to music, may also fulfil a person's unmet needs for self-expression, achievement, meaning, and purpose.

A within-subject design was used in this study wherein PtDD who underwent CMT intervention served as their own controls. While this methodology may not be ideal to generalize the study results to wider populations, it reduces the error variance associated with differences between subjects. By obviating group differences due to sampling error, it allows better sensitivity to the effects of CMT. To increase the internal validity, the period of observation between the control and intervention groups was kept consistent between 9:30 and 11:00 $\mathrm{h}$. Evenings were avoided to minimise the effect of 'sundowning'.

Some limitations of this pilot study are noteworthy. Although the study shows positive outcomes of CMT in improving positive engagement and emotions of PtDD during the period of engagement, it does not support any lasting effect of CMT beyond the sessions. The wellbeing of the subjects can be affected by intrinsic factors, such as fatigue and attention span, as well as extrinsic factors in the environment and treatment procedures. Hence, questions remain regarding the ideal duration of CMT and number of sessions per day. However, given the heterogeneous nature of PtDD, it is likely that uniform approaches will not suffice and a willingness to tailor care is necessary.

As direct observational assessments were employed, inter-rater differences always remain an issue despite efforts to minimize inconsistency by improving inter-rater reliability. As this study was primarily purposed to evaluate the effectiveness of an existing CMT 
programme for PtDD in an acute hospital ward, a within-subject design was employed with its known limitations in generalizability. Given the positive findings of the evaluation on a sample of a modest size, a subsequent study involving a larger sample and randomized controlled design with a more homogenous population is warranted.

\section{Conclusion}

As there has been little literature published hitherto on the effectiveness of music therapy for PtDD in acute hospital settings, the positive findings of this study are encouraging. Although the effects of CMT did not seem to extend beyond the period of music engagement, CMT can contribute to the overall well-being of PtDD if it is regularly scheduled into their daily routines or incorporated into other areas of care such as physical rehabilitation and nursing to increase patient compliance and cooperation.

\section{Disclosure Statement}

The authors declare that they have no conflicts of interest to disclose.

\section{References}

1 Phelan EA, et al: Association of incident dementia with hospitalizations. JAMA 2012;307:165-172.

-2 Fulop G, Strain JJ, Fahs MC, et al: A prospective study of the impact of psychiatric co-morbidity on length of stays of elderly medical-surgical inpatients. Psychosomatics 1998;39:273-280.

-3 Saravay SM, Lavin M: Psychiatric comorbidity and length of stay in the general hospital: a critical review of outcome studies. Psychosomatics 1994;35:233-252.

4 Bickel H, Cooper B, Wancata J: Psychiatric disorders in elderly general hospital patients: incidence and longterm prognosis (in German). Nervenarzt 1993;64:53-61.

5 Thompson F, Girling D, Green S, et al: Care of people with dementia in the general hospital; in Downs M, Bowers B (eds): Excellence in Dementia Care: Research into Practice. Berkshire, Open University Press, 2008, pp 301-318.

6 Saravay S, Kaplowitz M, Kurek J, et al: How do delirium and dementia increase length of stay of elderly general medical inpatients? Psychosomatics 2004;45:235-242.

7 Holmes J, House A: Psychiatric illness predicts poor outcome after hip fracture: a prospective cohort study. Psychol Med 2000;30:921-929.

8 Young J: Supporting health and physical well-being; in Downs M, Bowers B (eds): Excellence in Dementia Care: Research into Practice. Berkshire, Open University Press, 2008, pp 256-257.

-9 McCusker J, Cole M, Abrahamowicz M, et al: Delirium predicts 12-month mortality. Arch Intern Med 2002;162: 457-463.

10 González M, Martínez G, Calderón J, et al: Impact of delirium on short-term mortality in elderly inpatients: a prospective cohort study. Psychosomatics 2009;50:234-238.

11 Bradley L, Siddique CM, Dufron B: Reducing the use of physical restraints in long-term care facilities. J Gerontol Nurs 1995;21:21-34.

12 Evans D, Wood J, et al: Patient injury and physical restraint devices: a systematic review. J Adv Nurs 2003;41: 274-282.

13 O'Mahony R, Murthy L, Akunne A, et al: Synopsis of the National Institute for Health and Clinical Excellence guideline for prevention of delirium. Ann Intern Med 2011;154:746-751.

14 O'Hanlon S, O'Regan N, Meagher D, et al: Improving delirium care through early intervention: from bench to bedside to boardroom. J Neurol Neurosurg Psychiatry 2014;85:207-2013.

15 Sharon KI, Rudi GJW, Jane SS: Delirium in elderly people. Lancet 2014;383:911-922.

16 Cohen-Mansfield J, Dakheel-Ali M, Marx MS: Engagement in persons with dementia: the concept and its measurement. Am J Geriatric Psychiatry 2009;17:299-307.

$\checkmark 17$ Cohen-Mansfield J, Werner P: Management of verbally disruptive behaviours in the nursing home. J Gerantol Nurs 1997;24:10-17.

18 Hillecke T, Nickel A, Bolay HV: Scientific perspectives on music therapy. Ann NY Acad Sci 2005;1060:271-282.

19 McCaffrey R, Locsin R: The effect of music listening on acute confusion and delirium in elders undergoing elective hip and knee surgery. J Clin Nurs 2004;13:91-96. 
20 Chlan L: Effectiveness of a music therapy intervention on relaxation and anxiety for patients receiving ventilatory assistance. Heart Lung 1998;27:169-176.

21 Gold C, Voracek M, et al: Effects of music therapy for children and adolescents with psychopathology: a metaanalysis. J Child Psychol Psychiatry 2004;45:1054-1063.

22 Takahashi T, Matsushita H: Long-term effects of music therapy on elderly with moderate/severe dementia. J Music Ther 2006;43:317-333.

23 Vasionytė I, Madison G: Musical intervention for patients with dementia: a meta-analysis. J Clin Nurs 2013;22: 1203-1216.

24 Gerdner LA, Swanson EA: Effects of individualized music on confused and agitated elderly patients. Arch Psychiatr Nurs 1993;7:284-291.

25 Thomas DW, Heitman RJ, Alexander T: The effects of music on bathing cooperation for residents with dementia. J Music Ther 1997;34:246-259.

-26 Gerdner LA: Effects of individualized versus classical 'relaxation' music on the frequency of agitation in elderly persons with Alzheimer's disease and related disorders. Int Psychogeriatr 2000;12:49-65.

27 Ragneskog H, Asplund K, Kihlgren M, et al: Individualized music played for agitated patients with dementia: analysis of video-recorded sessions. Int J Nurs Pract 2001;7:146-155.

28 American Psychiatric Association: Diagnostic and Statistical Manual of Mental Disorders, ed 4. Washington, D.C., American Psychiatric Press, 1994.

29 Inouye S, van Dyck C, Alessi C, Balkin S, Siegal A, Horwitz R: Clarifying confusion: the confusion assessment method. Ann Int Med 1990;113:941-948.

-30 Judge KS, Camp CJ, et al: Use of Montessori-based activities for clients with dementia in adult day care: effects on engagement. Am J Alzheimers Dis Other Demen 2000;15:42-46.

31 Lawton MP, Van Haitsma K, Klapper J: Observed emotion rating scale. https://www.abramsoncenter.org/ media/1199/observed-emotion-rating-scale.pdf (accessed January 6, 2014).

-32 Lawton MP, Van Haitsma K, Klapper J: Observed affect in nursing home residents with Alzheimer's disease. J Gerontol B Psychol Sci Soc Sci 1996;51B:3-14.

-33 Johnson L, Deatrick EJ, et al: The use of music to improve exercise participation in people with dementia: a pilot study. Phys Occup Ther Geriatr 2012;30:102-108.

34 Sung HC, Chang AM: Use of preferred music to decrease agitated behaviours in older people with dementia: a review of the literature. J Clin Nurs 2005;14:1133-1140.

35 Baker F: The effects of live, taped and no music on people experiencing posttraumatic amnesia. J Music Ther 2002;38:82-104. 